\title{
Post-Segregational Killing, Altruistic Cell Death, and Other Toxin/Antitoxin Fallacies
}

\author{
Sooyeon Song and Thomas K. Wood \\ Now Published as: \\ Frontiers in Microbiology 9:814, 2018 \\ doi: 10.3389/fmicb.2018.00814
}

\begin{abstract}
Department of Chemical Engineering, Pennsylvania State University, University Park, Pennsylvania, 16802-4400, USA
\end{abstract}

*For correspondence. E-mail twood@engr.psu.edu;

Tel.(+)1 814-863-4811; Fax (1) 814-865-7846

Running title: Dubious toxin/antitoxin dogma

Keywords: toxin/antitoxin systems, persistence, growth inhibition, biofilm, stress response 


\begin{abstract}
The toxin/antitoxin (TA) field, born of controversy, is plagued by several prevailing misperceptions. For example, some TA systems stabilize plasmids and other genomic regions; however, the evidence of post-segregational killing by toxins of TA systems is weak. In addition, there are few credible reports of

5 cell death via TA systems like MazF/MazE and via phage exclusion systems. Although many aspects of the biological roles of TA systems remain enigmatic, there are now some clear, confirmed TA functions: (i) phage inhibition, (ii) plasmid maintenance, (iii) stress response (including regulation of loci distinct from the TA pair itself), (iv) biofilm formation, and (v) persistence. Therefore, this opinion piece aims to challenge the oft-repeated dogma related to TA systems with the goal of emphasizing their primary

10 biological role: constraining metabolism in a reversible manner. Hence, their roles in their five confirmed functions all stem from their ability to rapidly and reversibly reduce metabolic activity.
\end{abstract}

\title{
REVIEW
}

We recently elucidated several misperceptions in a field related to toxin/antitoxin (TA) systems, persister cell biology (Kim and Wood, 2016, 2017). Here, in the following sections, we aim to initiate a

15 similar discussion about several dubious yet entrenched theories that are related to TA systems. Most of these misperceptions are based on overproducing toxins; i.e., many toxins of TA systems appear lethal if produced from a strong promoter. However, conclusions based on overproducing proteins are seldom relevant for physiological conditions.

Nebulous nomenclature. Historically, TA systems were deemed cytosolic (not secreted); hence, TA

20 system toxins were considered primarily to affect the metabolism of the host that produced it rather than as serving as a weapon against other cells, such as colicins (Rendueles, et al., 2014). With a better understanding of their biological function, growth diminution as opposed to cell death, TA systems would be more aptly named "growth inhibitors" and "silencers of growth inhibitors," rather than "toxins," which imply a poison used against competitors rather than an internal means to reduce metabolism. However,

25 little would be served, and much confusion would ensue, if the name of the field was changed now.

Moreover, TA system components have now starting to be found outside the cell. For example, the 
type VII secretion system DNase toxin EsaD of the EsaD/EsaG TA system of Staphylococcus aureus (Cao, et al., 2016) is secreted for bacterial competitiveness; this is the second known toxin that is a DNase with RalR of the E. coli RalR/RalA TA system being the first (Guo, et al., 2014). The Xanthomonas oryzae type III secretion system toxin AvrRxo1 of the AvrRxo1/Arc1 TA system is also secreted into plants and modifies nicotinamide adenine dinucleotide. Additionally, antitoxin MqsA of the MqsR/MqsA TA system of Xylella fastidiosa (Santiago, et al., 2016) is secreted via outer membrane vesicles. Therefore, the distinction between external toxins like colicins and TA systems is becoming less clear.

Note that for TA pairs, we prefer the convention of listing the toxin first, then the antitoxin (e.g., $35 \mathrm{MazF} / \mathrm{MazE}$ ), regardless of the gene order. In addition, we prefer naming the antitoxin with four letters ending with an "A" (e.g., MqsA), where possible, to more readily identify the antitoxin.

How TA systems are classified is also evolving and is based on the mechanisms by with antitoxins mask toxin activity. There are six well-established systems (Page and Peti, 2016) that include type I (e.g., Hok/Sok), in which the antitoxin RNA prevents toxin mRNA translation (Gerdes, et al., 1986b), type II

40 (e.g., $\mathrm{CcdB} / \mathrm{CcdA}$ ), in which the antitoxin protein binds directly to the toxin to inhibit it (Ogura and Hiraga, 1983), type III (e.g., ToxN/ToxI), in which the antitoxin RNA binds the toxin to inhibit it (Fineran, et al., 2009a), type IV (e.g., CbtA/CbeA), in which the antitoxin protein interferes with the binding of the toxin with its target (Masuda, et al., 2012), type V (e.g., GhoT/GhoS), in which the protein antitoxin is an RNase that cleaves specifically the toxin mRNA (Wang, et al., 2012), and type VI (e.g.,

$45 \mathrm{SocB} / \mathrm{SocA}$ ), in which the antitoxin protein causes the degradation of the toxin (Aakre, et al., 2013). A type VII system (e.g., Hha/TomB) has also recently been discovered in which the antitoxin is an enzyme, but rather than cleaving toxin mRNA as in the type V system, the type VII antitoxin inactivates the toxin by oxidizing a cysteine residue (Marimon, et al., 2016).

Plasmid stabilization is not based on post-segregational killing. Toxin/antitoxin systems are

50 ubiquitous in that they are found in nearly all prokaryotic and archaeal genomes (Pandey and Gerdes, 2005). The first TA system, CcdB/CcdA (coupled cell division), was discovered in 1983, and was shown to maintain a mini-F plasmid (Ogura and Hiraga, 1983). In this seminal work, there was no mention of 
post-segregational killing or cell death, and toxin $\mathrm{CcdB}$ was shown to inhibit host cell division (Ogura and Hiraga, 1983).

55 The genesis of the idea of post-segregational killing arose with the report of the second TA system, Hok/Sok (host killing and suppressor of killing), that also stabilized a plasmid (R1) (Gerdes, et al., 1986a). In this report, that includes "Post-segregational killing of plasmid-free cells" in the title, the authors indicated, "we propose that the parB + locus mediates plasmid stability by killing cells that have lost the parB+ plasmid..." and claimed "irreversible killing of the host." Unfortunately, little evidence

60 was presented of cell death with physiologically-relevant levels of Hok toxin; in fact, the authors found a lack of cell lysis as demonstrated by a lack of extracellular $\beta$-galactosidase activity and only saw a decrease in cell viability when Hok toxin was produced by the strong lambda promoter (Gerdes, et al., 1986a). Furthermore, ghost cells were produced (but not a reduction in cell viability) with a copy number 8 plasmid construct harboring a cloned hok/sok locus (presumably under wild-type regulation but this is

65 not clear), and R1 plasmids (the physiological host harboring hok/sok) have a copy number of 6 (Ehrenberg and Sverredal, 1995). Later reports also lack cell killing with physiological-relevant amounts of Hok; i.e., killing is shown via overproduction of Hok (Gerdes, et al., 1986b). Hence, cell killing of plasmid-free hosts has not been demonstrated.

MazF does not cause programmed cell death. As a corollary to the lack of evidence for cell death in

70 plasmid maintenance studies outlined in the previous section, data indicating toxin MazF of the E. coli MazF/MazE TA system is responsible apoptotic behavior (i.e., programmed cell death, PCD) are also suspect. For example, although roughly $80 \%$ of vegetative Myxococcus xanthus cells undergo PCD during nutrient-limiting development (one of the few examples of bacterial PCD), it was claimed that MazF mediated this killing (Nariya and Inouye, 2008). However, this result was later shown to be an 75 artifact of a defect in the PilQ1 secretin of this strain used in the initial report by two different groups (Lee, et al., 2012, Boynton, et al., 2013). Hence, PCD does not occur for wild-type M. xanthus trains through MazF.

Similarly, it is doubtful that MazF is responsible for PCD in E. coli. PCD was claimed in 1996 for E. 
coli MazF (Aizenman, et al., 1996) and has been repeated frequently (Engelberg-Kulka and Glaser, 1999,

80 Sat, et al., 2001, Zhang, et al., 2003, Kumar, et al., 2016). Unfortunately, the initial claim of cell death was based on overproduction of MazF via a lambda $\mathrm{P}_{\mathrm{L}}$ promoter (Aizenman, et al., 1996). Furthermore, later studies from independent labs show PCD does not occur (Pedersen, et al., 2002, Tsilibaris, et al., 2007). Instead, MazF induces a reversible inhibition of growth (Pedersen, et al., 2002). Therefore, MazF at physiological levels does not cause PCD in any organism to date (Boynton, et al., 2013).

85 The extracellular death factor is unlikely to mediate cell death through MazF. The extracellular death factor (EDF, Asn-Asn-Trp-Asn-Asn) has been proposed as a quorum-sensing element for E. coli that mediates PCD via MazF (Kolodkin-Gal, et al., 2007). Unfortunately, it appears far more likely that this pentapeptide simply interacts with antibiotics extracellularly and has little to do with PCD or quorum sensing. Critically, EDF has been shown to scavenge free radicals (Gao, et al., 2010) and to protect cells

90 from ampicillin addition (Yan, et al., 2015). One of the main problems with the claim of quorum sensing via EDF is the necessity of adding rifampicin to see an effect (Kolodkin-Gal, et al., 2007); however, quorum-sensing systems do not, in general, require antibiotic pre-treatments to function, and the addition of rifampicin to inhibit transcription should activate many of the type I TA systems in E. coli in which the antitoxins are labile. Furthermore, no genes have been shown to be controlled by EDF nor has a protein

95 import or export system been elucidated; yet, quorum-sensing systems are based on secreted signals being either recognized by surface proteins or being imported and then affecting gene expression, like AI-2 (Pereira, et al., 2013) and indole (Lee, et al., 2015) in E. coli. Also, the protein from which EDF is supposed to be derived, Zwf, contains the sequence NNWDN, not NNWNN (Kumar, et al., 2016). Hence, many aspects of a quorum-sensing system have never been demonstrated for EDF. Since the previous 100 section outlines why MazF does not cause PCD, by the transitive property, it is doubtful that EDF mediates PCD through MazF (if PCD does not exist via MazF).

TA systems do not inhibit phage through altruistic killing. For the similar misperception that TA systems are involved in cell killing during phage inhibition, we must shoulder some of the blame. We first linked TA systems to phage propagation by discovering that the E. coli type I TA system Hok/Sok 
105 inhibits T4 phage propagation (Pecota and Wood, 1996); unfortunately, we also suggested the mechanism included altruistic killing. The role of TA systems in inhibiting phage propagation was verified eight years later using the type II MazF/MazE TA system to show a reduction in P1 phage propagation (Hazan and Engelberg-Kulka, 2004) and 13 years later with the type III ToxN/ToxI TA system (Fineran, et al., 2009b). Phage inhibition has also been reported for a likely type IV system, AbiEii/AbiEi from

110 Lactococcus lactis (Dy, et al., 2014). Therefore, phage inhibition has been shown for the four bestcharacterized types of TA systems. Additional proof of the role of TA systems in phage inhibition stems from the fact that phage carry antitoxins to combat host-encoded toxins (Otsuka and Yonesaki, 2012). So the role of TA systems in inhibiting phage propagation is solid.

However, in all these cases, cell death was not shown with a physiological dose of toxin. For 115 example, for ToxN/ToxI, even with a pBad promoter for toxin induction in E. coli, the authors found the TA system did not cause cell lysis and was reversible (Fineran, et al., 2009b); yet, this became apoptotic and beneficial behavior in later publications: "abortive infection, during which a bacteriophage-infected cell altruistically commits suicide to protect the clonal population, can be mediated by a TA pair" (Blower, et al., 2011). This misstatement was based on two references that do not indicate cell suicide but 120 instead show growth inhibition (Blower, et al., 2009, Fineran, et al., 2009b). To be clear, there may be some altruistic behavior in abortive infection systems based on other proteins, but cell death has not been shown to be mediated by TA systems. Therefore, it is most likely that the decrease in growth rate mediated by toxins (activated by phage attack) leads to phage inhibition.

Given this mechanism, one would also surmise that any protein that slows growth would inhibit 125 phage propagation (Wang and Wood, 2011). Hence, what makes TA systems interesting for phage inhibition is how they are induced by phage attack. In our original publication (Pecota and Wood, 1996), we proposed Hok/Sok was induced passively since T4 phage rapidly inhibits host transcription by modifying RNA polymerase, which leads to preferential degradation of the antitoxin RNA (Sok) and thereby activation of toxin Hok.

130 RelE and guanosine tetraphosphate (ppGpp) are not a TA system. Different from the heretofore 
undocumented claims of cell death from physiologically-relevant levels of toxins of TA systems, it was proposed that a metabolite, ppGpp, was a "toxin” and SpoT (ppGpp 3'-pyrophosphohydrolase) was an "antitoxin" (Amato, et al., 2013). This claim is dubious since ppGpp is merely the substrate of SpoT, so if this trend were continued, one could claim any metabolite that slows growth in excess along with a 135 protein that reduces its level as a TA pair. For example, following this logic, indole could be considered a "toxin" since it inhibits growth above $2 \mathrm{mM}$ and AcrEF could be considered an "antitoxin" since it exports indole (Lee, et al., 2015). Clearly this not appropriate. Even more perplexing is that ppGpp is not a toxin in that it serves to orchestrate the stringent response. Furthermore, the authors used carbon source transitions to form cells they called "persisters" but this, too, has been shown to be dubious (Kim and

140 Wood, 2017). Finally, their claim that cyclic adenosine monophosphate increases persistence (Amato, et al., 2013) was also found to be incorrect (Kwan, et al., 2015a).

There are clear biological roles for TA systems, including the stress response. Even this year it has been written that TA modules are "only weakly coupled to certain biological activities"(Harms, et al., 2018). This statement is dubious as there are several clear physiological roles of TA systems. For

145 example, the physiological roles of the MqsR/MqsA TA system are myriad (e.g., biofilm formation, stress response, global gene regulations, and persistence) and linked by oxidative stress. MqsR/MqsA of E. coli was discovered in 2004 via a whole-transcriptome study that identified the first TA system in biofilms (Ren, et al., 2004). The structure of the TA pair was used to discern that the toxin MqsR is an RNase (Brown, et al., 2009); by cleaving RNA with 5'-GCU sites independent of ribosomes (Yamaguchi, et al., 150 2009), MqsR can rapidly re-direct metabolism. In addition, MqsA was found to not only regulate its own promoter but to control the oxidative stress response (over 500 genes) by controlling sigma factor RpoS by binding to an upstream palindrome (Wang, et al., 2011); this result reduces biofilm formation indirectly by decreasing cyclic diguanylate (c-di-GMP) levels (Wang, et al., 2011). MqsA also controls biofilm directly by repressing the promoter of the curli regulator CsgD (Soo and Wood, 2013). Hence, 155 MqsA is a global regulator that serves to reduce biofilm formation in the absence of oxidative stress (by repressing rpoS and $c s g D$ ). 
Oxidative stress is experienced as the commensal strain E. coli encounters bile acid, so MqsR/MqsA helps the cell weather oxidative stress in the gastrointestinal tract (Kwan, et al., 2015b). Furthermore, the MqsR/MqsA TA system controls toxin/antitoxin system GhoT/GhoS by toxin MqsR cleaving 160 preferentially the mRNA of antitoxin GhoS, so during oxidative stress, MqsR activates another TA system as a cascade (Wang, et al., 2013); toxin MqsR cleaves all but 14 E. coli mRNAs and one of these 14 mRNAs that lacks a 5'-GCU site is GhoT. The result is that cell metabolism is reduced two ways: by (i) eliminating nearly all mRMA via toxin MqsR cleaving 5'-GCU sites and (ii) reducing ATP via toxin GhoT (Cheng, et al., 2014). When growth is extremely limited by this MqsR activity, the cell becomes 165 dormant and able to withstand extreme stress as evidenced by the fact that MqsR/MqsA is the first TA system that when deleted, reduces persistence (Kim and Wood, 2010) and MqsR increases persistence through GhoT (Wang, et al., 2013). Hence, there is a clear physiological role for the MqsR/MqsA TA system: it conveys the oxidative stress created by bile acid through MqsA derepressing the stress response of the cell (including increasing biofilm formation and persister cell formation) and through MqsR 170 differentially cleaving mRNA and activating GhoT.

This role of antitoxins controlling more than their own promoters was confirmed by antitoxin DinJ of the E. coli YafQ/DinJ TA system; DinJ binds and represses the promoter csgE (a positive regulator of RpoS mRNA translation) and thereby reduces the translation of the RpoS transcript (Hu, et al., 2012). Upon stress, antitoxin DinJ is degraded and RpoS translation is enhanced by CsgE which leads to less 175 motility, an increase in catalase activity, an increase in c-di-GMP, and an increase in biofilm formation. Antitoxins acting as global regulators extends to other strains as well; for example, in S. aureus, the SavS/SavR TA system silences the virulence genes hla and efb by binding their promoters (Wen, et al., 2018). Hence, the role of antitoxins as global regulators is established.

In addition, the TisB/IstR-1 type I TA system has been shown to have the physiological role of 180 producing persister cells when the cell is stressed by ciprofloxacin (Dörr, et al., 2010). Ciprofloxacin damages DNA which induces TisB toxin via an SOS response which results in TisB injuring the membrane thereby reducing the proton motive force and ATP levels. 
Conditional cooperativity is not prevalent. It is frequently indicated that conditional cooperativity is the prevalent form of type II TA system regulation (Harms, et al., 2018). The idea is that antitoxins can exist

185 as dimers and bind more than one toxin. At low ratios of toxin to antitoxin, the TA complex represses the TA promoter more than when the ratio of toxin to antitoxin is high. Although this concept may hold for some TA systems, there seem to be as many exceptions as there are those that fit this model. For example, the binding of MqsR toxin to MqsA causes the complex to fall off DNA since the binding sites of MqsA to DNA and to MqsR overlap, so MqsA cannot bind both its promoter and toxin. Hence, the regulation of mqsRA is not governed by conditional cooperativity (Brown, et al., 2013). Similarly, the E. coli YafQ/DinJ TA system (Ruangprasert, et al., 2014), the Yersinia pestis HicA3/HicB3 TA system (BibiTriki, et al., 2014), and the Proteus vulgaris HigB-HigA TA system (Schureck, et al., 2014) are all not regulated by conditional cooperativity.

\section{PERSPECTIVES}

195 Charles Darwin emphasized the importance of natural selection for beneficial mutations; i.e., changes that lead to improved reproduction will become dominant (Darwin, 1859). For bacteria, this is often oversimplified to imply organisms with fast growth will dominate. However, since most bacterial cells are starving (Schmidt, 2012), it appears it is just as relevant for microbes to elegantly decelerate their growth; i.e., the survival of the fittest doctrine should include selection of organisms with elegant diminution of

200 growth. This elegant growth diminution is the realm of TA systems, where they are clearly utilized to inhibit phage, stabilize genetic elements, activate the stress response, regulate biofilm formation, and generate persister cells. Hence, TA systems are nearly ubiquitous in procaryotes since they afford means to reduce growth elegantly. However, they are not utilized to kill cells.

\section{AUTHOR CONTRIBUTIONS}

205 TW conceived and wrote the manuscript. SS helped write the manuscript.

\section{ACKNOWLEDGEMENTS}

This work was supported by the Army Research Office (W911NF-14-1-0279) and funds derived from the 
Biotechnology Endowed Professorship at the Pennsylvania State University.

\section{REFERENCES}

Aakre, Christopher D., Phung, Tuyen N., Huang, D. and Laub, Michael T. (2013). A Bacterial Toxin Inhibits DNA Replication Elongation through a Direct Interaction with the $\beta$ Sliding Clamp. Mol Cell 52, 617-628. doi http://dx.doi.org/10.1016/j.molcel.2013.10.014

Aizenman, E., Engelberg-Kulka, H. and Glaser, G. (1996). An Escherichia coli chromosomal "addiction module" regulated by guanosine 3',5'-bispyrophosphate: a model for programmed bacterial cell death. Proceedings of the National Academy of Sciences 93, 6059-6063. doi

Amato, Stephanie M., Orman, Mehmet A. and Brynildsen, Mark P. (2013). Metabolic Control of Persister Formation in Escherichia coli. Molecular Cell 50, 475-487. doi

Bibi-Triki, S., Li de la Sierra-Gallay, I., Lazar, N., Leroy, A., Van Tilbeurgh, H., Sebbane, F., et al. (2014). Functional and Structural Analysis of HicA3-HicB3, a Novel Toxin-Antitoxin System of Yersinia pestis. Journal of Bacteriology 196, 3712-3723. doi 10.1128/jb.01932-14

Blower, T. R., Fineran, P. C., Johnson, M. J., Toth, I. K., Humphreys, D. P. and Salmond, G. P. C. (2009). Mutagenesis and Functional Characterization of the RNA and Protein Components of the toxIN Abortive Infection and Toxin-Antitoxin Locus of Erwinia. Journal of Bacteriology 191, 60296039. doi 10.1128/jb.00720-09

Blower, T. R., Pei, X. Y., Short, F. L., Fineran, P. C., Humphreys, D. P., Luisi, B. F., et al. (2011). A processed noncoding RNA regulates an altruistic bacterial antiviral system. Nat Struct Mol Biol 18, 185-190. doi http://www.nature.com/nsmb/journal/v18/n2/abs/nsmb.1981.html\#supplementaryinformation

Boynton, T. O., McMurry, J. L. and Shimkets, L. J. (2013). Characterization of Myxococcus xanthus MazF and implications for a new point of regulation. Molecular Microbiology 87, 1267-1276. doi $10.1111 / \mathrm{mmi} .12165$

Brown, B. L., Lord, D. M., Grigoriu, S., Peti, W. and Page, R. (2013). The E. coli toxin MqsR destabilizes the transcriptional repression complex formed between the antitoxin MqsA and the mqsRA operon promoter. Journal of Biological Chemistry 288, 1286-1294. doi 10.1074/jbc.M112.421008

Brown, B. L., Grigoriu, S., Kim, Y., Arruda, J. M., Davenport, A., Wood, T. K., et al. (2009). Three dimensional structure of the MqsR:MqsA complex: A novel toxin:antitoxin pair comprised of a toxin homologous to RelE and an antitoxin with unique properties. PLoS Pathog. 5, e1000706. doi

Cao, Z., Casabona, M. G., Kneuper, H., Chalmers, J. D. and Palmer, T. (2016). The type VII secretion system of Staphylococcus aureus secretes a nuclease toxin that targets competitor bacteria. Nature Microbiology 2, 16183. doi 10.1038/nmicrobiol.2016.183 
Cheng, H.-Y., Soo, V. W. C., Islam, S., McAnulty, M. J., Benedik, M. J. and Wood, T. K. (2014). Toxin GhoT of the GhoT/GhoS toxin/antitoxin system damages the cell membrane to reduce adenosine triphosphate and to reduce growth under stress. Environmental Microbiology 16, 1741-1754. doi $10.1111 / 1462-2920.12373$

Darwin, C. (1859) On the Origin of Species. London, United Kingdom: John Murray.

Dörr, T., Vulić, M. and Lewis, K. (2010). Ciprofloxacin causes persister formation by inducing the TisB toxin in Escherichia coli. PLoS Biol. 8, e1000317. doi

Dy, R. L., Przybilski, R., Semeijn, K., Salmond, G. P. C. and Fineran, P. C. (2014). A widespread bacteriophage abortive infection system functions through a Type IV toxin-antitoxin mechanism. Nucleic Acids Research 42, 4590-4605. doi 10.1093/nar/gkt1419

Ehrenberg, M. and Sverredal, A. (1995). A Model for Copy Number Control of the Plasmid R1. Journal of Molecular Biology 246, 472-485. doi https://doi.org/10.1006/jmbi.1994.0099

Engelberg-Kulka, H. and Glaser, G. (1999). Addiction Modules and Programmed Cell Death and Antideath in Bacterial Cultures. Annual Review of Microbiology 53, 43-70. doi 10.1146/annurev.micro.53.1.43

Fineran, P. C., Blower, T. R., Foulds, I. J., Humphreys, D. P., Lilley, K. S. and Salmond, G. P. C. (2009a). The phage abortive infection system, ToxIN, functions as a protein-RNA toxin-antitoxin pair. Proc Natl Acad Sci U S A 106, 894-899. doi 10.1073/pnas.0808832106

Fineran, P. C., Blower, T. R., Foulds, I. J., Humphreys, D. P., Lilley, K. S. and Salmond, G. P. C. (2009b). The phage abortive infection system, ToxIN, functions as a protein-RNA toxin-antitoxin pair. Proceedings of the National Academy of Sciences 106, 894-899. doi 10.1073/pnas.0808832106

Gao, Y., Chen, K., Zhang, B., Li, X., Chen, L., Li, Y., et al. (2010). Antioxidant and free radicalscavenging activity of the extracellular death factor in Escherichia coli. Peptides 31, 1821-1825. doi https://doi.org/10.1016/j.peptides.2010.06.034

Gerdes, K., Rasmussen, P. B. and Molin, S. (1986a). Unique Type of Plasmid Maintenance Function: Postsegregational Killing of Plasmid-Free Cells. Proceedings of the National Academy of Sciences 83, 3116-3120. doi

Gerdes, K., Bech, F. W., Jorgensen, S. T., Lobner-Olesen, A., Rasmussen, P. B., Atlung, T., et al. (1986b). Mechanism of Postsegregational Killing by the hok gene Product of the parB System of Plasmid R1 and its Homology with the RelF Gene Product of the E. coli relB Operon. The European Molecular Biology Organization Journal 5, 2023-2029. doi

Guo, Y., Quiroga, C., Chen, Q., McAnulty, M. J., Benedik, M. J., Wood, T. K., et al. (2014). RalR (a DNase) and RalA (a small RNA) form a type I toxin-antitoxin system in Escherichia coli. Nucleic Acids Research 42, 6448-6462. doi 10.1093/nar/gku279 
Harms, A., Brodersen, D. E., Mitarai, N. and Gerdes, K. (2018). Toxins, Targets, and Triggers: An Overview of Toxin-Antitoxin Biology. Molecular Cell, on-line. doi https://doi.org/10.1016/j.molcel.2018.01.003

Hazan, R. and Engelberg-Kulka, H. (2004). Escherichia coli mazEF-mediated cell death as a defense mechanism that inhibits the spread of phage P1. Mol. Genet. Genomics 272, 227-234. doi

Hu, Y., Benedik, M. J. and Wood, T. K. (2012). Antitoxin DinJ influences the general stress response through transcript stabilizer CspE. Environmental Microbiology 14, 669-679. doi 10.1111/j.14622920.2011.02618.x

Kim, J.-S. and Wood, T. K. (2016). Persistent Persister Misperceptions. Frontiers in Microbiology 7, 2134. doi $10.3389 /$ fmicb.2016.02134

Kim, J.-S. and Wood, T. K. (2017). Tolerant, Growing Cells from Nutrient Shifts Are Not Persister Cells. mBio 8, e00354-00317. doi 10.1128/mBio.00354-17

Kim, Y. and Wood, T. K. (2010). Toxins Hha and CspD and small RNA regulator Hfq are involved in persister cell formation through MqsR in Escherichia coli. Biochem. Biophys. Res. Commun. 391, 209-213. doi

Kolodkin-Gal, I., Hazan, R., Gaathon, A., Carmeli, S. and Engelberg-Kulka, H. (2007). A Linear Pentapeptide Is a Quorum-Sensing Factor Required for mazEF-Mediated Cell Death in Escherichia coli. Science 318, 652-655. doi 10.1126/science.1147248

Kumar, S., Kolodkin-Gal, I., Vesper, O., Alam, N., Schueler-Furman, O., Moll, I., et al. (2016). Escherichia coli Quorum-Sensing EDF, A Peptide Generated by Novel Multiple Distinct Mechanisms and Regulated by trans-Translation. mBio 7doi 10.1128/mBio.02034-15

Kwan, B. W., Osbourne, D. O., Hu, Y., Benedik, M. J. and Wood, T. K. (2015a). Phosphodiesterase DosP increases persistence by reducing cAMP which reduces the signal indole. Biotechnology and Bioengineering 112, 588-600. doi 10.1002/bit.25456

Kwan, B. W., Lord, D. M., Peti, W., Page, R., Benedik, M. J. and Wood, T. K. (2015b). The MqsR/MqsA Toxin/Antitoxin System Protects Escherichia coli During Bile Acid Stress. Environ. Microbiol. 17, 3168-3181. doi 10.1111/1462-2920.12749

Lee, B., Holkenbrink, C., Treuner-Lange, A. and Higgs, P. I. (2012). Myxococcus xanthus Developmental Cell Fate Production: Heterogeneous Accumulation of Developmental Regulatory Proteins and Reexamination of the Role of MazF in Developmental Lysis. Journal of Bacteriology 194, 30583068. doi $10.1128 / \mathrm{jb} .06756-11$

Lee, J.-H., Wood, T. K. and Lee, J. (2015). Roles of Indole as an Interspecies and Interkingdom Signaling Molecule. Trends in Microbiology 23, 707-718. doi https://doi.org/10.1016/j.tim.2015.08.001

Marimon, O., Teixeira, J. M. C., Cordeiro, T. N., Soo, V. W. C., Wood, T. L., Mayzel, M., et al. (2016). An oxygen-sensitive toxin-antitoxin system. Nat Commun 7, 13634. doi 10.1038/ncomms13634 
Masuda, H., Tan, Q., Awano, N., Wu, K.-P. and Inouye, M. (2012). YeeU enhances the bundling of cytoskeletal polymers of MreB and FtsZ, antagonizing the CbtA (YeeV) toxicity in Escherichia coli. Mol Microbiol 84, 979-989. doi 10.1111/j.1365-2958.2012.08068.x

Nariya, H. and Inouye, M. (2008). MazF, an mRNA Interferase, Mediates Programmed Cell Death during Multicellular Myхососcus Development. Cell 132, 55-66. doi https://doi.org/10.1016/j.cell.2007.11.044

Ogura, T. and Hiraga, S. (1983). Mini-F plasmid genes that couple host cell division to plasmid proliferation. Proc. Natl. Acad. Sci. USA 80, 4784-4788. doi

Otsuka, Y. and Yonesaki, T. (2012). Dmd of bacteriophage T4 functions as an antitoxin against Escherichia coli LsoA and RnlA toxins. Molecular Microbiology 83, 669-681. doi 10.1111/j.13652958.2012.07975.x

Page, R. and Peti, W. (2016). Toxin-antitoxin systems in bacterial growth arrest and persistence. Nature Chemical Biology 12, 208. doi 10.1038/nchembio.2044

Pandey, D. P. and Gerdes, K. (2005). Toxin-antitoxin loci are highly abundant in free-living but lost from host-associated prokaryotes. Nucleic Acid Res 33, 966-976. doi

Pecota, D. C. and Wood, T. K. (1996). Exclusion of T4 Phage by the hok/sok Killer Locus from Plasmid R1. J. Bacteriol. 178, 2044-2050. doi

Pedersen, K., Christensen, S. and Gerdes, K. (2002). Rapid induction and reversal of a bacteriostatic condition by controlled expression of toxins and antitoxins. Mol Microbiol 45, 501 - 510. doi

Pereira, C. S., Thompson, J. A. and Xavier, K. B. (2013). AI-2-mediated signalling in bacteria. FEMS Microbiology Reviews 37, 156-181. doi 10.1111/j.1574-6976.2012.00345.x

Ren, D., Bedzyk, L. A., Thomas, S. M., Ye, R. W. and Wood, T. K. (2004). Gene expression in Escherichia coli biofilms. Appl Microbiol Biotechnol 64, 515-524. doi doi: 10.1007/s00253-003$1517-y$

Rendueles, O., Beloin, C., Latour-Lambert, P. and Ghigo, J.-M. (2014). A new biofilm-associated colicin with increased efficiency against biofilm bacteria. The Isme Journal 8, 1275 . doi 10.1038/ismej.2013.238

Ruangprasert, A., Maehigashi, T., Miles, S. J., Giridharan, N., Liu, J. X. and Dunham, C. M. (2014). Mechanisms of Toxin Inhibition and Transcriptional Repression by Escherichia coli DinJ-YafQ. Journal of Biological Chemistry 289, 20559-20569. doi 10.1074/jbc.M114.573006

Santiago, A. d. S., Mendes, J. S., Santos, C. A. d., Toledo, M. A. S. d., Beloti, L. L., Crucello, A., et al. (2016). The Antitoxin Protein of a Toxin-Antitoxin System from Xylella fastidiosa Is Secreted via Outer Membrane Vesicles. Frontiers in Microbiology 7doi 10.3389/fmicb.2016.02030 
Sat, B., Hazan, R., Fisher, T., Khaner, H., Glaser, G. and Engelberg-Kulka, H. (2001). Programmed Cell Death in Escherichia coli: Some Antibiotics Can Trigger mazEFLethality. Journal of Bacteriology 183, 2041-2045. doi 10.1128/jb.183.6.2041-2045.2001

Schmidt, T. M. (2012) Bacteria Battling for Survival. In Microbes and Evolution: American Society of Microbiology.

Schureck, M. A., Maehigashi, T., Miles, S. J., Marquez, J., Cho, S. E., Erdman, R., et al. (2014). Structure of the Proteus vulgaris HigB-(HigA)2-HigB Toxin-Antitoxin Complex. Journal of Biological Chemistry 289, 1060-1070. doi 10.1074/jbc.M113.512095

Soo, V. W. C. and Wood, T. K. (2013). Antitoxin MqsA Represses Curli Formation Through the Master Biofilm Regulator CsgD. Sci. Rep. 3, 3186. doi 10.1038/srep03186

Tsilibaris, V., Maenhaut-Michel, G., Mine, N. and Van Melderen, L. (2007). What Is the Benefit to Escherichia coli of Having Multiple Toxin-Antitoxin Systems in Its Genome? J. Bacteriol. 189, 6101-6108. doi 10.1128/jb.00527-07

Wang, X. and Wood, T. K. (2011). Toxin-antitoxin systems influence biofilm and persister cell formation and the general stress response. Appl Environ Microbiol 77doi 10.1128/aem.05068-11

Wang, X., Lord, D. M., Hong, S. H., Peti, W., Benedik, M. J., Page, R., et al. (2013). Type II toxin/antitoxin MqsR/MqsA controls type $\mathrm{V}$ toxin/antitoxin GhoT/GhoS. Environmental Microbiology 15, 1734-1744. doi 10.1111/1462-2920.12063

Wang, X., Kim, Y., Hong, S. H., Ma, Q., Brown, B. L., Pu, M., et al. (2011). Antitoxin MqsA helps mediate the bacterial general stress response. Nat. Chem. Biol. 7, 359-366. doi

Wang, X., Lord, D. M., Cheng, H. Y., Osbourne, D. O., Hong, S. H., Sanchez-Torres, V., et al. (2012). A new type $\mathrm{V}$ toxin-antitoxin system where mRNA for toxin GhoT is cleaved by antitoxin GhoS. Nat Chem Biol 8, 855-861. doi 10.1038/Nchembio.1062

Wen, W., Liu, B., Xue, L., Zhu, Z., Niu, L. and Sun, B. (2018). Autoregulation and virulence control by the toxin-antitoxin system SavRS in Staphylococcus aureus. Infection and Immunity doi:10.1128/IAI.00032-18, on-line. doi 10.1128/iai.00032-18

Yamaguchi, Y., Park, J.-H. and Inouye, M. (2009). MqsR, a crucial regulator for quorum sensing and biofilm formation, is a GCU-specific mRNA interferase in Escherichia coli. J. Biol. Chem. 284, 28746-28753. doi 10.1074/jbc.M109.032904

Yan, Z., Li, G., Gao, Y., Zhai, W., Qi, Y. and Zhai, M. (2015). The extracellular death factor (EDF) protects Escherichia coli by scavenging hydroxyl radicals induced by bactericidal antibiotics. SpringerPlus 4, 182. doi 10.1186/s40064-015-0968-9

Zhang, Y., Zhang, J., Hoeflich, K. P., Ikura, M., Qing, G. and Inouye, M. (2003). MazF Cleaves Cellular mRNAs Specifically at ACA to Block Protein Synthesis in Escherichia coli. Molecular Cell 12, 913923. doi http://dx.doi.org/10.1016/S1097-2765(03)00402-7 